\title{
Diabetic uterus environment may play a key role in alterations of DNA methylation of several imprinted genes at mid-gestation in mice
}

Zhao-Jia Ge ${ }^{1,2,3}$, Qiu-Xia Liang ${ }^{3}$, Shi-Ming Luo ${ }^{3}$, Yan-Chang Wei ${ }^{3}$, Zhi-Ming Han ${ }^{3}$, Heide Schatten ${ }^{4}$, Qing-Yuan Sun ${ }^{3^{*}}$ and Cui-Lian Zhang ${ }^{1 *}$

\begin{abstract}
Background: Maternal diabetes mellitus not only has severe deleterious effects on fetal development, but also it affects transmission to the next generation. However, the underlying mechanisms for these effects are still not clear.

Methods: We investigated the methylation patterns and expressions of the imprinted genes Peg3, Snrpn, and H19 in mid-gestational placental tissues and on the whole fetus utilizing the streptozotocin (STZ)-induced hyperglycemic mouse model for quantitative analysis of methylation by PCR and quantitative real-time PCR. The protein expression of Peg3 was evaluated by Western blot.

Results: We found that the expression of $\mathrm{H} 19$ was significantly increased, while the expression of Peg 3 was significantly decreased in dpc10.5 placentas of diabetic mice. We further found that the methylation level of Peg3 was increased and that of $\mathrm{H} 19$ was reduced in dpc10.5 placentas of diabetic mice. When pronuclear embryos of normal females were transferred to normal/diabetic (NN/ND) pseudopregnant females, the methylation and expression of Peg3 in placentas was also clearly altered in the ND group compared to the NN group. However, when the pronuclear embryos of diabetic female were transferred to normal pesudopregnant female mice (DN), the methylation and expression of Peg3 and H19 in dpc10.5 placentas was similar between the two groups.
\end{abstract}

Conclusions: We suggest that the effects of maternal diabetes on imprinted genes may primarily be caused by the adverse uterus environment.

Keywords: Maternal diabetes milieu, DNA imprinting, Placenta

\section{Background}

Infants of mothers with pre-existing types 1,2 or gestational diabetes have significantly higher rates of perinatal mortality and major congenital anomaly [1,2]. Diabetic embryopathy can affect many organ systems including the heart and the neural tube [3-5]. The effects of maternal diabetes mellitus on fetal development have been studied in various animal models. In drug-induced animal models, embryo development was disturbed by maternal hyperglycaemia [6,7]. In non-obese diabetic (NOD) mouse models

\footnotetext{
* Correspondence: sunqy@ioz.ac.cn; luckyzcl@qq.com

${ }^{3}$ State Key Laboratory of Reproductive Biology, Institute of Zoology, Chinese Academy of Sciences, Beijing 100101, P. R. China

'Reproductive Medicine Center, Henan Provincial People's Hospital, Zhengzhou 450003, Henan Province, P. R. China

Full list of author information is available at the end of the article
}

of spontaneous type 1 diabetes mellitus, only $20 \%$ of recovered NOD embryos reached the blastocyst stage at 96 hours of superovulation compared with $90 \%$ of embryos recovered from nondiabetic animals [6].

Several reports have shown that teratogenesis may be a direct result of hyperglycemia [8,9]. Previous studies found that expressions of genes related to metabolism, development and signal transduction were altered in embryos by the diabetic environment [10,11]. Moley et al. showed that the mRNA and protein expression of the glucose transporters GLUT-1, GLUT-2, and GLUT-3 were decreased in embryos from streptozotocin (STZ)induced hyperglycaemic mice [12]. These results partly elucidate how maternal diabetes mellitus causes abnormal embryo development. But it is still not clear how the adverse effects are inherited to the next generations. 
Wellen et al. found that excessive glucose affected histone acetylation via the citrate lyase pathway [13]. This finding suggests the possibility that the epigenome of the embryo may contribute significantly to abnormal fetal development in diabetic females. DNA methylation, one of the epigenetic modifications on DNA, can regulate relative gene expression, $\mathrm{X}$-chromosome inactivation, as well as genomic imprinting [14]. Genomic imprinting includes the formation of DNA methylation at specific loci in a parent-of-origin-specific manner [15]. If DNA methylation on imprinted genes is not acquired/maintained properly, embryonic development and the offspring's health would be affected $[16,17]$.

The DNA methylation pattern of imprinted genes is susceptible to being affected by the environment [18]. Several reports have shown that preimplantation culture and manipulation can cause an abnormal methylation status of Differentially Methylated Regions (DMRs) at imprinted loci and these changes may induce abnormal fetal development [19-21]. If maternal nutrients are altered the DNA methylation patterns may also be changed and this will induce abnormalities during fetal development. During gestation, if female rats are fed with cholinedeficient diets, the DNA methylation of G9a and Suv39h1 is mis-regulated [22]. These data indicate that the adverse maternal environment exerts adverse effects on DNA methylation during genomic imprinting establishment and maintenance.

We hypothesized that impaired DNA methylation at imprinted loci may play a key role in causing abnormal embryo development in maternal diabetes mellitus. In our lab, we have found that the DNA methylation patterns in DMRs of imprinted genes Peg3, Snrpn and H19 in oocytes was not altered by maternal diabetes at 15 days of injection of STZ [23], but the embryonic development was affected. This indicated that the uterus environment may have deleterious effects on embryonic development. We examined the methylation patterns of DMRs of Peg3, Snrpn, and H19 in day post-coitum (dpc)10.5 placenta and fetus in the STZ-induced mouse model. We found that the expression and methylation levels of the imprinted genes were altered by maternal diabetes mellitus in placentas at $10.5 \mathrm{dpc}$ of gestation. Previous studies have shown that if the pregestational type 1 diabetes mellitus was cured at prepregnancy, the risks of adverse pregnancy outcomes was reduced in women [24]. In animal models, if diabetic females were treated with insulin, embryonic development was not significantly different from that in non-diabetic females [6]. Therefore, we also investigated whether the adverse effects caused by maternal diabetes on imprinted genes in placentas could be corrected by embryo transfer.

\section{Methods}

\section{Ethics statement}

All procedures described were reviewed and approved by the ethical committee of the Institute of Zoology, Chinese Academy of Sciences.

All mice were provided by the Beijing Vital River Experimental Animals Centre and fed in a temperature controlled room with a light cycle of 12 L: 12D (light: dark).

\section{Generation of the diabetic mouse model}

Female $\mathrm{CD}-1^{\circ}$ (strain code; 022) mice, aged 6-7 weeks, received a single intraperitoneal injection of streptozotocin (STZ) at a dose of $230 \mathrm{mg} / \mathrm{kg}$ [25]. Four days later, blood glucose levels were checked using a glucometer, Blood Testing Equipment, Accu-CHEK Active (Roche Diagnostic, Germany). If glucose levels were higher than $17.0 \mathrm{mmol} / \mathrm{l}$, the mice were selected and used as the diabetic model (diabetic mice $n=53$ ). Mice of similar age injected with buffer were selected as control (non-diabetic mice $n=49$ ).

\section{Collecting placentas and fetuses at mid-gestation}

Diabetic/nondiabetic mice were mated naturally with normal male mice within 15 days of STZ/buffer injection and were determined to be pregnant when the vaginal plug was examined at $0.5 \mathrm{~d}$. At $10.5 \mathrm{~d}$ of gestation, placentas (trophoblast population) and whole fetuses were collected. Samples were frozen immediately in liquid nitrogen and stored at $-80^{\circ} \mathrm{C}$ until used.

\section{DNA purification and quantitative analysis of methylation by PCR (qAMP)}

DNA was purified from one half of the bisected fetus or placentas using DNA Tissue Kit (Tiangen, China) following the manufacturer's directions. To investigate the methylation conditions, qAMP was conducted as described by Oakes et al. and Lopes et al. [26,27]. Briefly, fetal or placental DNA ( 1.5 $\mu \mathrm{g} /$ enzyme $)$ was digested with methylation-sensitive (HhaI, New England Biolabs, Beijing, China; HpaII, New England Biolabs, Beijing, China) and methylation-dependent (McrBc, New England Biolabs, Beijing, China) restriction enzymes, respectively. The resulting product was used as template for real-time PCR reaction using the SYBR green kit (Kangwei Inc., China). The qAMP was performed employing a Rotor-Gene Q Real-Time PCR instrument (Qiagen). The percentage of methylation for cytosinephosphate-guanine $(\mathrm{CpG})$ sites was based on $\triangle \mathrm{Ct}$ (cycle threshold values between digested and undigested sham aliquots). For HhaI, the percent methylation is 100 
$\left(\mathrm{e}^{-0.7(\Delta \mathrm{Ct})}\right)$; for $M c r B C$, the percent methylation is 100 $\left(1-\mathrm{e}^{-0.7(\Delta \mathrm{Ct})}\right)$. The primers described in Table 1 were designed to span $\sim 180 \mathrm{bps}$ of the DMRs of the maternally methylated sequences: Peg3 and Snrpn, as well as the paternally methylated $H 19$.

\section{RNA isolation and mRNA expression assays}

RNA was extracted from the other half of the bisected fetus and placentas using RNA Tissue kit (Tiangen, China) according to the manufacturer's instructions. cDNA first-strand synthesis was performed using Superscript II (Invitrogen). The first-strand cDNA was used as template and the expressions of Peg3, Snrpn and H19 were evaluated by quantitative real-time PCR (qRT-PCR) using Rotor-gen Q (Qiagen, Germany). Triple reactions were analyzed for each sample and the threshold cycle value was normalized to the housekeeping gene of peptidylprolyl isomerase A (Ppia). The primers are shown in Table 1. The expression levels were calculated using the $2^{-\Delta \Delta \mathrm{Ct}}$ method.

\section{Western blot and immunohistochemistry analysis}

Placental proteins ( $n=6$ from 4 litters) were extracted from cell lysis and quantified for western blot analysis as previously described [28]. Briefly, proteins were heated for $5 \mathrm{~min}$ at $100^{\circ} \mathrm{C}$. Then proteins were separated by SDS-PAGE and electrically transferred to polyvinylidene fluoride membranes. After that, the membranes were blocked in TBST containing 5\% skimmed milk for $2 \mathrm{~h}$, followed by incubation overnight at $4^{\circ} \mathrm{C}$ with rabbit antipeg3 (1:500, Bioss, China, code: bs-1870R). After washed with TBST, membranes were incubated with goat antirabbit IgG $(1: 1000)$ for $1 \mathrm{~h}$ at $37^{\circ} \mathrm{C}$. Beta-actin was used as loading control.

For immunohistochemistry analysis, fresh placentas ( $n=6$ from 4 litters) were fixed in $4 \%$ paraformaldehyde overnight at $4^{\circ} \mathrm{C}$ and washed in $50 \%$ ethanol for 1 hour at room temperature. After that, samples were stored in $70 \%$ ethanol at $4^{\circ} \mathrm{C}$ until used. The fixed samples were dehydrated in a graded ethanol series, cleared in xylene, and embedded in paraffin wax. The embedded placentas were sectioned at $8 \mu \mathrm{m}$ and incubated with rabbit anti-Peg3 (1:500, Bioss, China, code: bs-1870R) overnight at $4^{\circ} \mathrm{C}$. The samples were then incubated with the biotin-labeled secondary antibody for 30 minutes. Staining was carried out using the Vectastain ABC kit and $\mathrm{DAB}$ peroxidase substrate kit (Vector Laboratories, Burlingame, CA).

\section{Embryo transfer}

To obtain pronuclear stage embryos from diabetic and nondiabetic mice, the estrous female diabetic/ non-diabetic mice were naturally mated with normal males, respectively. Vaginal plug was examined the next morning. The mice with a vaginal plug were killed and the pronuclear embryos were collected from their oviductal ampullae.

To transfer embryos to pseudopregnant mice which were produced by mating the normal estrous female mice with males with vasoligation, the pesudopregnant mice were anesthetized by intraperitoneal injection of sodium pentobarbital (40 mg/kg, Sigma). Then eight embryos were transferred into the oviduct of a pseudopregnant mouse. The mice were fed until gestational $10.5 \mathrm{dpc}$ and then killed. Embryos and placentas were collected.

\section{Statistical analysis}

Data are presented as means \pm SD. The methylation level of DMRs and expression levels of genes from different groups were compared by independent-sample t-test. A probability level of $\mathrm{P}<0.05$ was considered significant.

\section{Results}

Methylation patterns of imprinted genes' DMRs in $10.5 \mathrm{dpc}$ placentas are affected by maternal diabetes mellitus

To understand how maternal diabetes mellitus affects methylation levels of imprinted genes in $10.5 \mathrm{dpc}$ placentas from female diabetic mice mated with normal males

Table 1 Oligonucleotides and annealing temperature utilized for qAMP and qRT-PCR of imprinted genes

\begin{tabular}{|c|c|c|c|}
\hline & & \multicolumn{2}{|c|}{ Primer sequence } \\
\hline & & Forward & Reverse \\
\hline \multirow[t]{4}{*}{ qAMP } & $H 19$ & 5'-AGCCGTTGTGAGTGGAAAGA-3' & 5'-CATAGCGGCTTCGGACATT-3' \\
\hline & Snrpn & 5'-CTCCTCAGAACCAAGCGTCT-3' & 5'-ATTCCGGTCAGAGGGACAGA-3' \\
\hline & Peg3 & 5'-GGTGTCCCGCAGCCCTTG-3' & 5'-CGGAGCACAGCACTCTACGC-3' \\
\hline & chr9:106724005-106724149 & 5'-GATCTATTCCTTCCTITACTIT-3' & 5'-TCCTGGGAAATGAAGTIT-3' \\
\hline Peg3 & qRT-PCR & 5'-TTGGACTGGACAGAGATGATGACA-3' & 5'-ATTCTGGTATGACTCGGCATCCT-3' \\
\hline Snrpn & & 5'-AGGCCCATCCCAGCAGGTCAT-3' & 5'-GCGGGTACTGGGTTGGGGCTC-3' \\
\hline H19 & & 5'- CTTGTCGTAGAAGCCGTCTGTTC-3' & 5'- GTAGCACCATTTCTTTCATCTTGAGG-3' \\
\hline Ppia & & 5'- CGCGTCTCCTTCGAGCTGTTTG-3' & 5'- TGTAAAGTCACCACCCTGGCACAT-3' \\
\hline
\end{tabular}


within 15 days of STZ injection, qAMP was used to evaluate the average methylation levels of DMRs. For paternally imprinted gene $H 19$, the average methylation levels were $43.92 \pm 3.24 \%$ and $52.5 \pm 1.42 \%$ in $M c r B c$ sites $(P=0.021)$, and $46.21 \pm 2.06 \%$ and $45.34 \pm 1.8 \%$ in HhaI sites in diabetic and nondiabetic groups (Figure 1A), respectively. At HhaI/HpaII sites, the mean methylation levels of Snrpn were $52.62 \pm 2.44 \%, 39.44 \pm 2.45 \%$ in the diabetic group and $47.32 \pm 1.52 \%, 36.21 \pm 1.81 \%$ in controls (Figure 1B), respectively. In placentas, the methylation levels of DMRs of maternally imprinted gene Peg3 were significantly altered in diabetic mothers compared to controls. As shown in Figure $1 \mathrm{C}$, the average methylation levels of Peg3 in $M c r B c$ and HpaII sites were $62.66 \pm 2.33 \%, 53.74 \pm 2.61 \%$ and $53.82 \pm 1.91 \%, 44.94 \pm 1.49 \%$ in diabetic and nondiabetic groups, respectively.

To confirm that qAMP is sensitive enough to evaluate DNA methylation, we have chosen a DNA fragment in which all CpG sites were unmethylated. We determined that qAMP was sensitive enough to detect the DNA methylation status for imprinted gene DMRs (Figure 1D).

The average methylation levels of imprinted genes are not altered in the fetus of diabetic females

To investigate the methylation status of imprinted genes in dpc10.5 fetus, the methylation levels in DMRs of maternally imprinted genes Peg3, Snrpn, and paternally imprinted gene $H 19$ were determined by qAMP. The methylation pattern in DMR of H19 was not affected by maternal diabetes in the fetus of female diabetic mice (Figure 2A). There was also no significant difference observed in the methylation patterns of Snrpn DMR between the diabetic and non-diabetic embryos (Figure $2 \mathrm{~B}$ ). The average methylation level of Peg3 was similar between diabetic and nondiabetic groups when the samples were digested by HpaII and $M c r B c$ (Figure 2C).
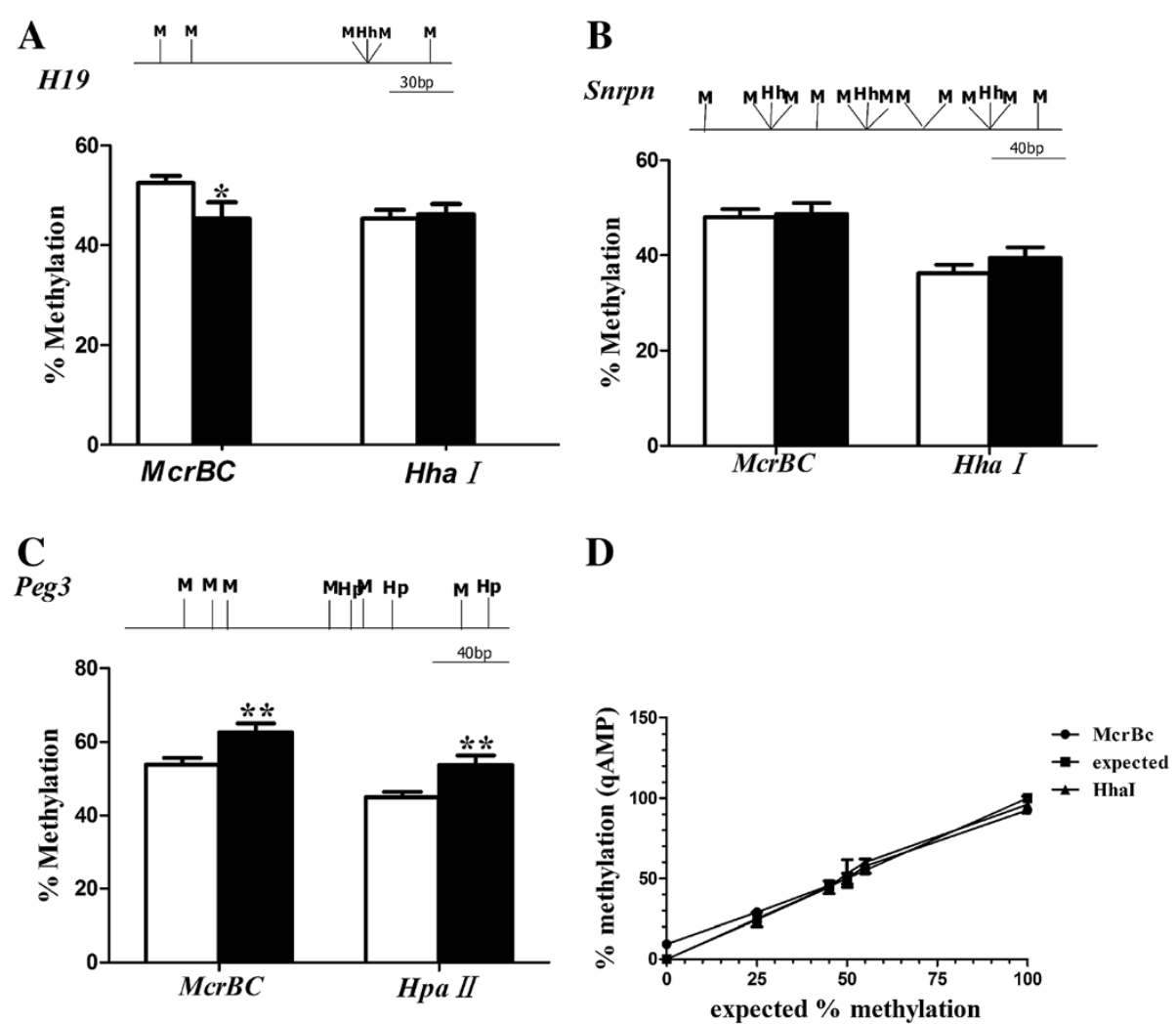

Figure 1 Quantitative DNA methylation in DMRs of imprinted genes in 10.5dpc placentas as revealed by qAMP. Diabetic ( $n=26$ from 5 litters) and nondiabetic ( $n=25$ from 5 litters) placentas at mid-gestation were recovered. (A) The paternally methylated H19; (B, C) Percentage methylation values at single and groups of restriction sites in the maternally methylated DMRs of Snrpn and Peg3. The lines represent the DMRs which were analyzed and the letter indicates the recognition sites of the enzymes. M, McrBc; Hh, Hhal; Hp, Hpall. (D) Confirmation of gene methylation level was determined by qAMP to expected values. Hhal and McrBc sites in chr9:106724005-106724149 are unmethylated in the genome. A primer pair flanking these sites was utilized to amplify DNA (NIH 3 T3 mouse genomic DNA and CpG methylated NIH 3 T3 mouse genomic DNA, NEB) mixed at the ratios: 100:0, 75:25, 55:45, 50:50, 45:55, 0:100. The qAMP values were close to expected values. Data are presented as mean $\pm \mathrm{SE}$ for each enzyme employed. White bar, non-diabetic group; black bar, diabetic group; ${ }^{* P}<0.05$, **P $<0.01$. 


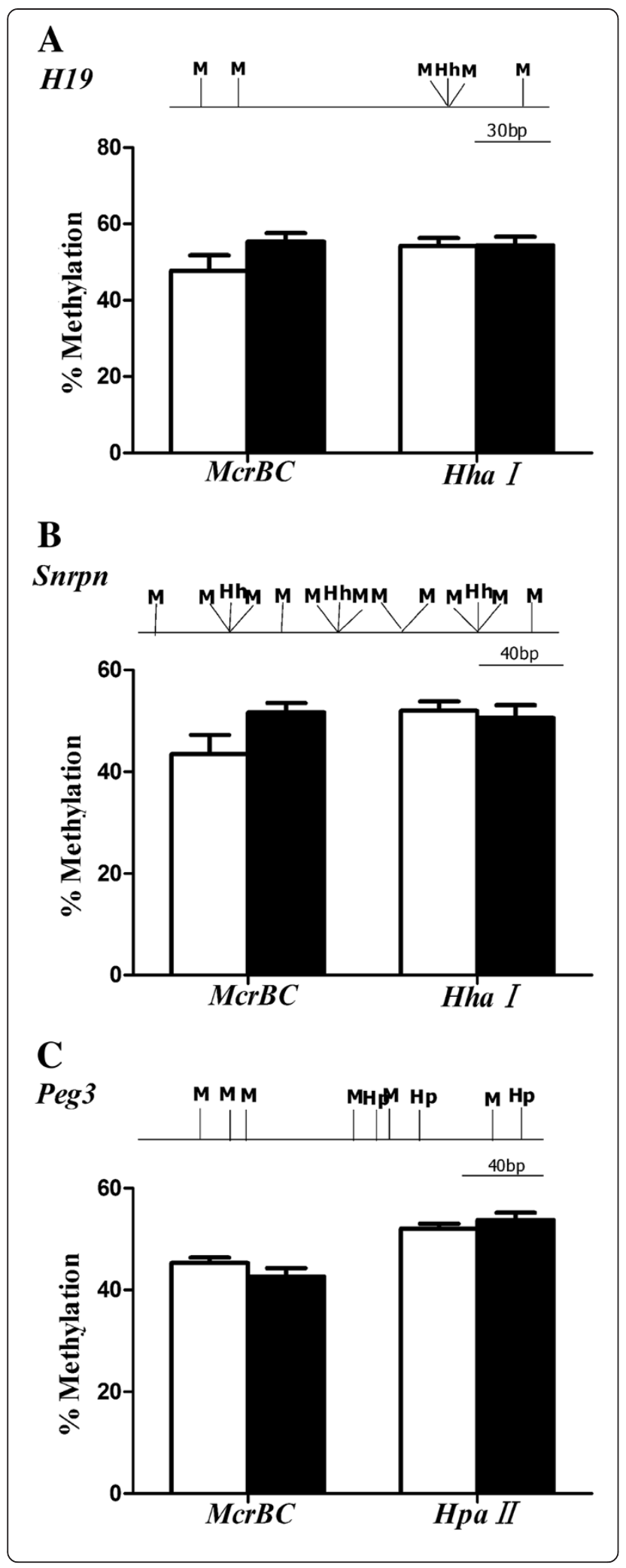

Figure 2 Average DNA methylation levels in DMRs of imprinted genes in $10.5 \mathrm{dpc}$ fetus as revealed by qAMP. Diabetic ( $n=12$ from 4 litters) and control ( $n=12$ from 4 litters) fetus were collected at $10.5 \mathrm{dpc}$ of gestation. DNA was digested with Hhal (Hh), Hpall(Hp) or McrBC (M) and amplified using real-time PCR. The locations of flanked restriction sites were displayed for each DMR. (A) Shown are the average methylation levels of paternally methylated gene $\mathrm{H} 19$ DMR; $(\mathbf{B}, \mathbf{C})$ represented the DNA methylation status in DMRs of Snrpn and Peg3. Data were presented as mean \pm SE for each enzyme employed. White bar, non-diabetic group; black bar, diabetic group.

Expressions of Peg3 and $\mathrm{H} 19$ are altered by maternal diabetes in placentas but not in the fetus at mid-gestation

We also assessed whether the expression levels of imprinted genes were affected by maternal diabetes in placentas and fetus at $10.5 \mathrm{dpc}$ by quantitative real-time PCR (qRT-PCR). For placentas, the expression levels of Snrpn were similar between the two groups, indicating that the expression was not disturbed by maternal diabetes mellitus. But expression levels of paternally imprinted gene $H 19$ and maternally imprinted gene Peg3 showed significant alterations in the diabetic group compared to controls (Figure 3A). In the fetus, the expression levels of Snrpn, Peg3 and H19 in the diabetic groups were similar to controls (Figure 3B).

We also investigated the protein expression of Peg3 in placentas. The result showed that the protein level was significantly lower $(\mathrm{P}=0.039)$ in the diabetic group than in the control group (Figure 3C, D). And we found that Peg3 was expressed in the placenta (Figure 3E) and the expression level in the control group was clearly higher than that in the diabetic group.

Alterations of Peg3 methylation and expression in the dpc10.5 placenta are observed in different litters

We also investigated the methylation level and mRNA expression of Peg3 in dpc10.5 placentas between different diabetic mothers. Six litters marked as s1-s6, respectively, were analyzed. For s2, s3 and s6, methylation levels at $\mathrm{McrBc}$ and HpaII were significantly higher compared to the nondiabetic mother (Figure 4A, B). The methylation levels of s1 and s4 were clearly higher at HpaII than the control (Figure 4B). At $M c r B c$, the methylation level of s5 was increased in dpc10.5 placentas of diabetic females (Figure 4A). Meanwhile, we found that the expression of Peg3 was significantly reduced by maternal diabetes mellitus for s2, s3, s5 and s6 (Figure 4C).

The alterations of Peg3 methylation and expression may mainly be caused by the adverse uterus environment When pronuclear embryos of normal mothers were transferred to diabetic/normal pseudopregnant females (ND/NN), we found that the methylation level of Peg3 was increased in ND (Figure 5A) placentas. Meanwhile, 


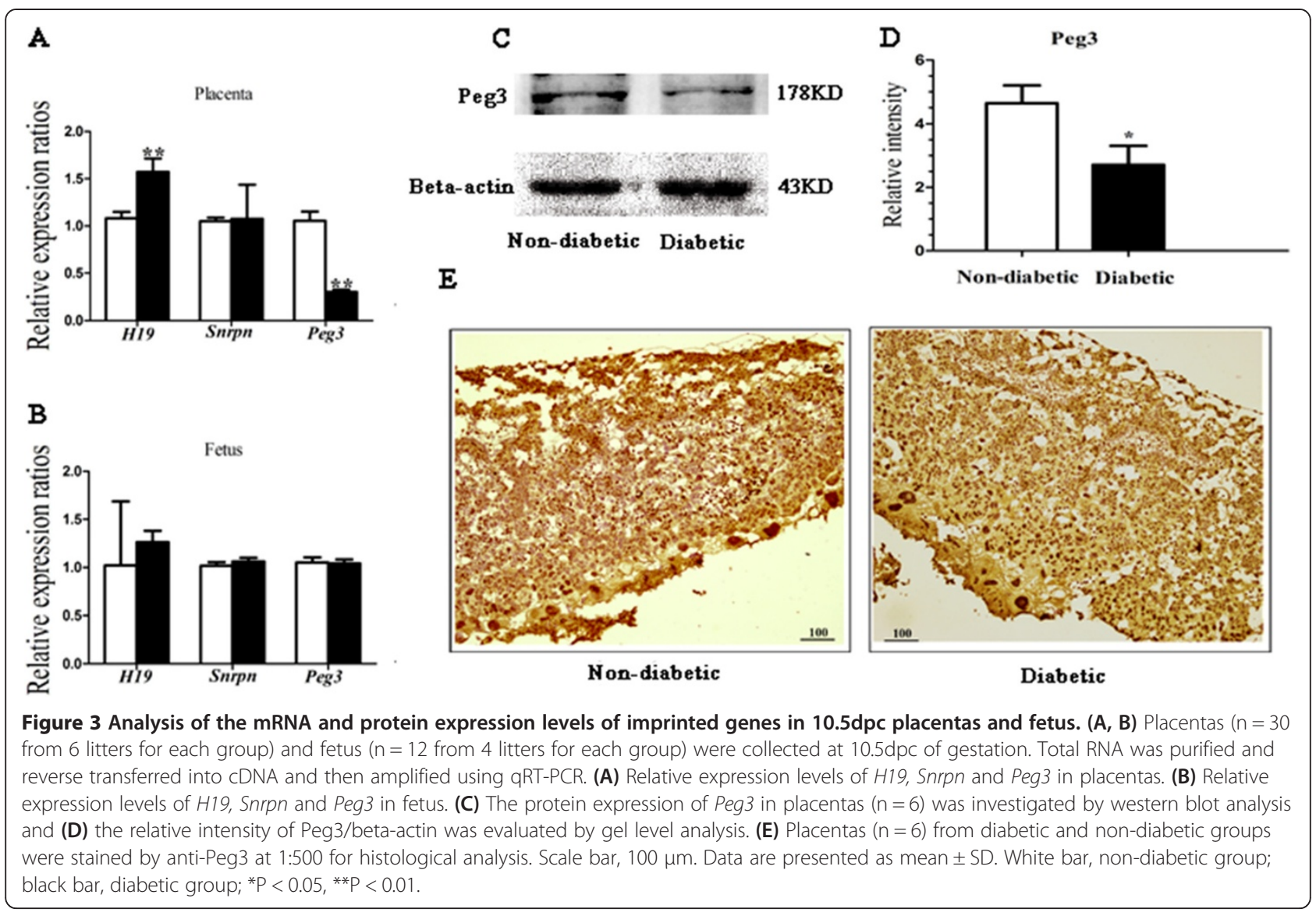

we observed that the mRNA expression of Peg3 was reduced in ND $(\mathrm{P}<0.01$, Figure $5 \mathrm{~B})$ placentas.

When we transferred pronuclear embryos from diabetic/non-diabetic mice to normal pseudopregnant mice $(\mathrm{DN} / \mathrm{NN})$, the average methylation levels in DMRs of Peg3 were similar between $\mathrm{DN}$ and $\mathrm{NN}$ in placentas (Figure 5C). The expression of Peg3 in placentas of NN and DN was also similar (Figure 5D).

\section{Discussion}

Proper establishment and maintenance of DNA methylation patterns are crucial for embryo development and survival. In the present study, we investigated whether maternal diabetes mellitus could perturb the acquisition/ maintenance of DNA methylation during embryo development. In our study, we found that the expressions of Peg3 and H19 were altered in placentas at $10.5 \mathrm{dpc}$; we also identified evident changes in the methylation level in the DMRs of them. Strikingly, these effects were not observed when diabetic pronuclear embryos were transferred into non-diabetic recipients.

The placenta is not only the specialized organ used by the developing embryo and fetus to obtain nutrients and oxygen from the mother, but it also acts as a key selectively permeable barrier between the mother and the fetus [29]. In pregnancies complicated by pre-gestational diabetes (type 1 and type 2), the de novo synthesis of blood vessels in the placenta shows abnormalities [30]. Several studies show that the expressions of leptin, leptin receptors, androgen receptor and peroxisome proliferator-activated receptors (PPARs) are altered by maternal diabetes mellitus in placentas $[31,32]$. In our study, we also observed that the mRNA levels of $H 19$ and Peg3 were significantly altered in placentas from diabetic females compared to controls. These imprinted genes are expressed monoallelically in a parent-of-origin specific manner. They are generally located in clusters, epigenetically marked by DNA methylation on differentially methylated regions (DMRs) which regulate relative genes' expression [33-35]. Interestingly, there were significant changes of average methylation levels in DMRs of $H 19$ and Peg3 in placentas from diabetic mice. It is well known that DNA methylation is a mechanism regulating expression of imprinted genes. Our results indicate that abnormal methylation levels may be a reason for the changed expressions of these genes. However, these changes might be induced by different distribution of Peg3 in different placental cell populations. Hiby et al. found that Peg3 was expressed in the trophoblast of the developing placenta at 9 days post-coitum (d.p.c.) and the trophoblast populations of the well-developed placenta at $15 \mathrm{dpc}$ [36]. In our study, 


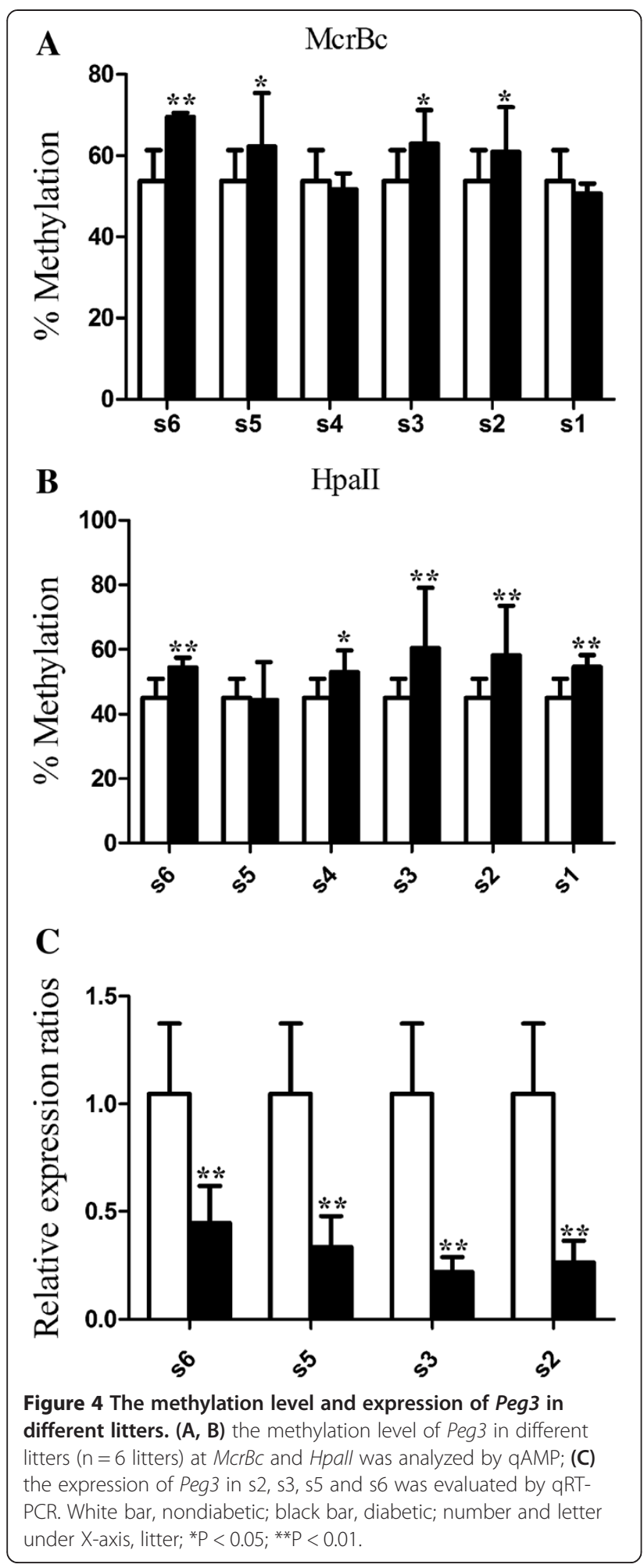

the placenta was separated and used to carry out all the relative assays. By immunohistochemistry analysis, we observed that all the trophoblast populations were stained. The Western blot analysis also indicated that the protein level of Peg3 in diabetic placentas was obviously lower.
These findings might partly elucidate how maternal diabetes mellitus induces abnormal embryo development, because proper DNA methylation patterns are important for placenta and fetus development.

Previous studies found that the diabetic condition is detrimental to pre- and post-implantation embryo development. Congenital malformations are approximately 34 times more frequent in infants from diabetic mothers than from non-diabetic mothers [37,38]. In both chemically induced and spontaneous diabetic models, significant abnormalities in pre-implantation embryo development have been observed $[6,39,40]$. But the DNA methylation patterns in fetus from diabetic females are still unclear. In our study, we examined the expression and average methylation levels of some imprinted genes in the placenta and embryo at $10.5 \mathrm{dpc}$. We found that the expression of relative imprinted genes was not altered in diabetic dpc10.5 fetus compared with controls. The expression of $H 19$ was consistent with that reported by Shao et al. [41]. The average methylation levels in DMRs of them were also similar to controls. Although another study found that the methylation level of H19Igf2 imprint control region was increased in the embryonic day 14 fetus from diabetic mice [41], we found that the methylation level of H19 DMR was not affected by maternal diabetes in the $10.5 \mathrm{dpc}$ embryo. Different methods employed in these studies may be a reason for the difference in results. Another reason may relate to the fact that we selected different fragments as targets. Because severe loss of DNA methylation in imprinted genes causes embryo absorption and miscarriage during early development, it was not possible to identify significant loss of DNA methylation in DMRs of imprinted genes in live fetus from diabetic females. Another reason may be the protective function of the placenta which serves as filter between mother and fetus. In our study, we found no changes of imprinted genes in fetus from mothers with diabetes mellitus, but the expression and methylation levels were significantly affected by maternal diabetes in placentas. These findings suggest that maternal diabetes may affect fetus development by altering placental functions.

Studies have shown that if maternal diabetes mellitus is well controlled, the deleterious effects on embryo development can be partially corrected. Studies also have shown that when the diabetic females are treated with insulin, embryo development is not significantly different from non-diabetic females [6]. These results indicate that the diabetic uterus environment may play a key role in abnormal embryonic development. So we transferred pronuclear embryos of normal females to normal/diabetic pseudopregnant females and found that the DNA methylation patterns and expression of Peg3 in dpc10.5 placentas were significantly changed by the diabetic 

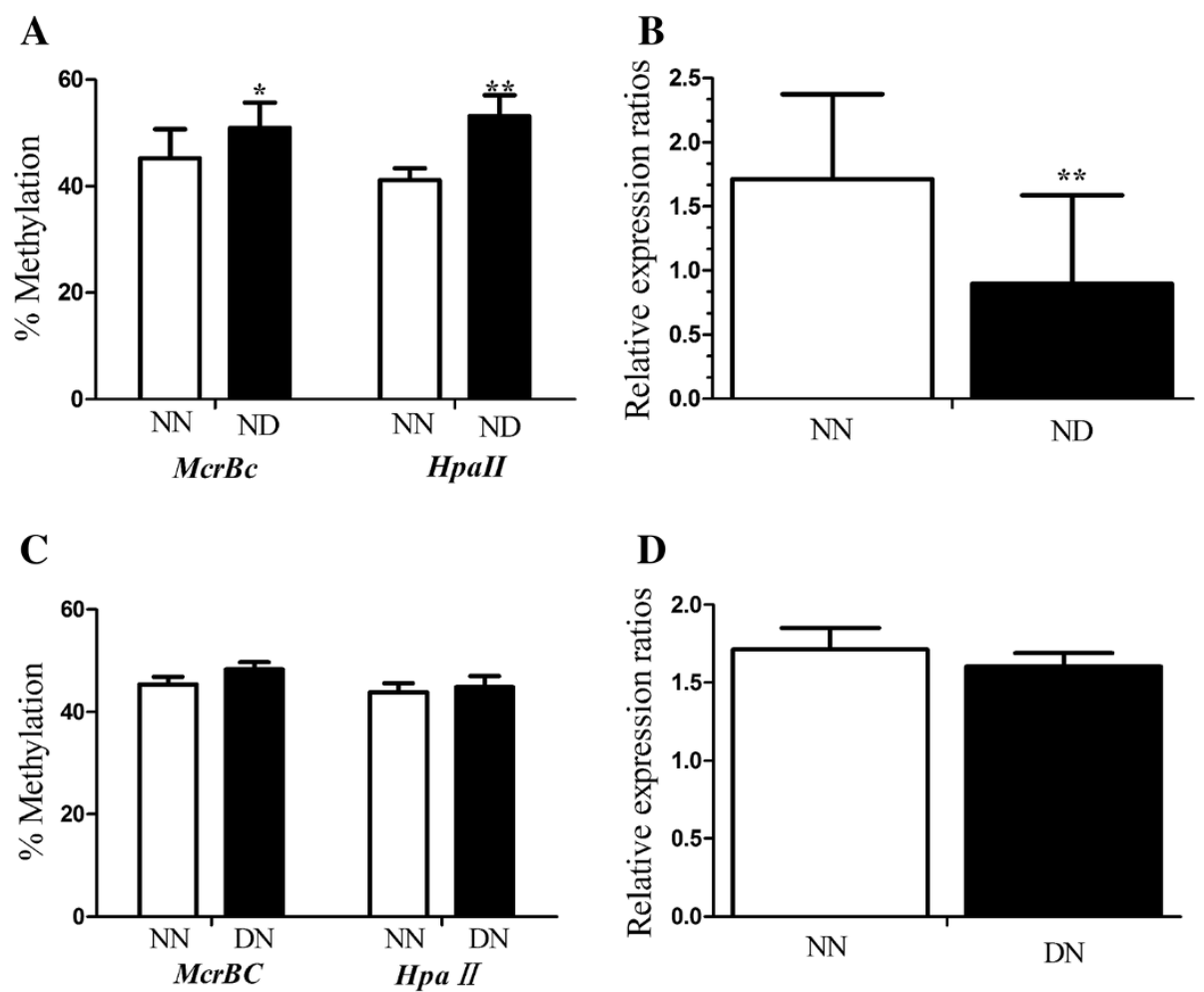

Figure 5 DNA methylation and expression levels of Peg3 in placentas at mid-gestation. (A, B) Non-diabetic pronuclear embryos were transferred to normal/diabetic (NN/ND) pseudopregnant female and the DNA methylation and expression levels of Peg3 ( $n, N N: N D=12: 15)$ in 10.5d placentas were analyzed by qAMP and qRT-PCR, respectively. (A) methylation level of Peg3 in dpc10.5 placenta; (B) expression of Peg3 in placenta. (C, D) DN's ( $n=15$ from 4 litters) and NN's ( $n=15$ from 4 litters) placentas were collected at mid-gestation. DNA was digested with Hpallor McrBC and amplified using real-time PCR. (C) the methylation in DMRs of Peg3 in placentas; (D) Relative expression levels of Peg3 in placentas. Data were presented as mean \pm SD. ${ }^{*} P<0.05,{ }^{* * P}<0.01$.

uterus environment. However, while pronuclear embryos of diabetic mothers were transferred to normal pseudopregnant females $(\mathrm{DN})$, the expression and methylation levels of $H 19$ and Peg3 in placentas were similar between $\mathrm{DN}$ and NN. Another study has demonstrated that these genes' methylation status in oocytes of diabetic females was not affected [23] at 15 days of injection of STZ. So we propose that the altered expression and methylation levels of imprinted genes may primarily be caused by the adverse uterus environment of diabetic females and it could be corrected by embryo transfer.

\section{Conclusions}

In summary, our data show that the expression of imprinted genes is disturbed by maternal diabetes mellitus in mid-gestation and this may be caused by altered methylation patterns in DMRs. Such effect could be corrected by transfer of pronuclear embryos from a diabetic mother into a normal uterus. In addition, when pronuclear embryos of normal females were transferred to diabetic pseudopregnant females, the alterations of imprinted genes were also observed. Therefore, the detrimental maternal diabetic uterus environment may play a key role in causing the deleterious effects on imprinted genes in the dpc10.5 placenta. Our evaluations of methylation and expression of imprinted genes were only carried out in live mid-gestation embryos and several DMRs. The conditions regarding the entire genomic imprinting pattern still need further clarification.

\section{Competing interests}

The authors declare that they have no competing interests.

\section{Authors' contributions}

ZJG carried out the epigenetic analysis, designed the assay and wrote the manuscript. QXL carried out the epigenetic analysis. SML, YCW and ZJG carried out PCR amplification and epigenetic analysis. HZM participated in analyzing the data. HS participated in revising the manuscript. QYS and CLZ participated in the design of the assay and revising the manuscript. All authors read and approved the final manuscript.

\section{Acknowledgements}

We thank Yi Hou (Institute of Zoology, Chinese Academy of Sciences), and Ying-Chun Ouyang (Institute of Zoology, Chinese Academy of Sciences) for technical assistance.

\section{Author details}

${ }^{1}$ Reproductive Medicine Center, Henan Provincial People's Hospital, Zhengzhou 450003, Henan Province, P. R. China. ${ }^{2}$ Reproductive Medicine Center, People's Hospital of Zhengzhou University, Zhengzhou 450003, Henan Province, P. R. China. ${ }^{3}$ State Key Laboratory of Reproductive Biology, 
Institute of Zoology, Chinese Academy of Sciences, Beijing 100101, P. R. China. ${ }^{4}$ Department of Veterinary Pathobiology, University of Missouri, Columbia, MO 65211, USA

Received: 13 September 2013 Accepted: 26 December 2013 Published: 30 December 2013

\section{References}

1. Yang J, Cummings EA, O'Connell C, Jangaard K: Fetal and neonatal outcomes of diabetic pregnancies. Obstet Gynecol 2006, 108:644-650.

2. Kousseff BG: Gestational diabetes mellitus (class A): a human teratogen? Am J Med Genet 1999, 83:402-408.

3. Loffredo CA, Wilson PD, Ferencz C: Maternal diabetes: an independent risk factor for major cardiovascular malformations with increased mortality of affected infants. Teratology 2001, 64:98-106.

4. Cleves MA, Hobbs CA: Collaborative strategies for unraveling the complexity of birth defects. J Matern Fetal Neona 2004, 15:35-38.

5. Kucera J: Rate and type of congenital anomalies among offspring of diabetic women. J Reprod Med 1971, 7:73-82.

6. Moley KH, Vaughn WK, DeCherney AH, Diamond MP: Effect of diabetes mellitus on mouse pre-implantation embryo development. J Reprod Fertil 1991, 93:325-332.

7. Lea RG, McCracken JE, McIntyre SS, Smith W, Baird JD: Disturbed development of the preimplantation embryo in the insulin-dependent diabetic BB/E rat. Diabetes 1996, 45:1463-1470.

8. Baker L, Piddington R: Diabetic embryopathy: a selective review of recent trends. J Diabetes Complications 1993, 7:204-212.

9. Corrigan N, Brazil DP, McAuliffe F: Fetal cardiac effects of maternal hyperglycemia during pregnancy. Birth Defects Res A Clin Mol Teratol 2009, 85:523-530.

10. Phelan SA, Ito M, Loeken MR: Neural tube defects in embryos of diabetic mice: role of the Pax-3 gene and apoptosis. Diabetes 1997, 46:1189-1197.

11. Wentzel P, Wentzel CR, Gareskog MB, Eriksson UJ: Induction of embryonic dysmorphogenesis by high glucose concentration, disturbed inositol metabolism, and inhibited protein kinase C activity. Teratology 2001, 63:193-201.

12. Moley KH, Chi MM, Mueckler MM: Maternal hyperglycemia alters glucose transport and utilization in mouse preimplantation embryos. Am J Physiol 1998, 275:E38-47.

13. Wellen KE, Hatzivassiliou G, Sachdeva UM, Bui TV, Cross JR, Thompson CB: ATP-citrate lyase links cellular metabolism to histone acetylation. Science 2009, 324:1076-1080.

14. Bestor TH: The DNA methyltransferases of mammals. Hum Mol Genet 2000, 9:2395-2402

15. Reik W, Dean W, Walter J: Epigenetic reprogramming in mammalian development. Science 2001, 293:1089-1093.

16. Bourc'his D, Xu GL, Lin CS, Bollman B, Bestor TH: Dnmt3L and the establishment of maternal genomic imprints. Science 2001, 294:2536-2539.

17. Hata K, Okano M, Lei H, Li E: Dnmt3L cooperates with the Dnmt3 family of de novo DNA methyltransferases to establish maternal imprints in mice. Development 2002, 129:1983-1993.

18. Zhang TY, Labonte B, Wen XL, Turecki G, Meaney MJ: Epigenetic mechanisms for the early environmental regulation of hippocampal glucocorticoid receptor gene expression in rodents and humans. Neuropsychopharmacology 2013, 38:111-123.

19. Nagy A, Rossant J, Nagy R, Abramow-Newerly W, Roder JC: Derivation of completely cell culture-derived mice from early-passage embryonic stem cells. Proc Natl Acad Sci U S A 1993, 90:8424-8428.

20. Moore T, Reik W: Genetic conflict in early development: parental imprinting in normal and abnormal growth. Rev Reprod 1996, 1:73-77.

21. Dean W, Bowden L, Aitchison A, Klose J, Moore T, Meneses JJ, Reik W, Feil R: Altered imprinted gene methylation and expression in completely ES cell-derived mouse fetuses: association with aberrant phenotypes. Development 1998, 125:2273-2282.

22. Davison JM, Mellott TJ, Kovacheva VP, Blusztajn JK: Gestational choline supply regulates methylation of histone $\mathrm{H} 3$, expression of histone methyltransferases G9a (Kmt1c) and Suv39h1 (Kmt1a), and DNA methylation of their genes in rat fetal liver and brain. J Biol Chem 2009, 284:1982-1989.
23. Ge ZJ, Liang XW, Guo L, Liang QX, Luo SM, Wang YP, Wei YC, Han ZM, Schatten H, Sun QY: Maternal diabetes causes alterations of DNA methylation statuses of some imprinted genes in murine oocytes. Biol Reprod 2013, 88:117.

24. Pearson DWM, Kernaghan D, Lee R, Penney GC, Scottish Diabetes in Pregnancy Study G: The relationship between pre-pregnancy care and early pregnancy loss, major congenital anomaly or perinatal death in type I diabetes mellitus. BJOG 2007, 114:104-107.

25. Wang Q, Ratchford AM, Chi MMY, Schoeller E, Frolova A, Schedl T, Moley $\mathrm{KH}$ : Maternal diabetes causes mitochondrial dysfunction and meiotic defects in murine oocytes. Mol Endocrinol 2009, 23:1603-1612.

26. Oakes CC, La Salle S, Robaire B, Trasler JM: Evaluation of a quantitative DNA methylation analysis technique using methylation-sensitive/ dependent restriction enzymes and real-time PCR. Epigenetics 2006, 1:146-152.

27. Lopes FL, Fortier AL, Darricarrere N, Chan D, Arnold DR, Trasler JM: Reproductive and epigenetic outcomes associated with aging mouse oocytes. Hum Mol Genet 2009, 18:2032-2044.

28. Huang L, Wang ZB, Jiang ZZ, Hu MW, Lin F, Zhang QH, Luo YB, Hou Y, Zhao $Y$, Fan HY, et al: Specific disruption of Tsc1 in ovarian granulosa cells promotes ovulation and causes progressive accumulation of corpora lutea. PLoS One 2013, 8:e54052.

29. Leach L: Placental vascular dysfunction in diabetic pregnancies: intimations of fetal cardiovascular disease? Microcirculation 2011 18:263-269.

30. Leach L, Babawale MO, Anderson M, Lammiman M: Vasculogenesis, angiogenesis and the molecular organisation of endothelial junctions in the early human placenta. J Vasc Res 2002, 39:246-259.

31. Uzelac PS, Li X, Lin J, Neese LD, Lin L, Nakajima ST, Bohler H, Lei Z: Dysregulation of leptin and testosterone production and their receptor expression in the human placenta with gestational diabetes mellitus. Placenta 2010, 31:581-588.

32. Kurtz M, Capobianco E, Martinez N, Fernandez J, Higa R, White V, Jawerbaum A: Carbaprostacyclin, a PPARdelta agonist, ameliorates excess lipid accumulation in diabetic rat placentas. Life Sci 2010, 86:781-790.

33. Holmes R, Soloway PD: Regulation of imprinted DNA methylation. Cytogenet Genome Res 2006, 113:122-129.

34. Lewis A, Reik W: How imprinting centres work. Cytogenet Genome Res 2006, 113:81-89.

35. Fauque P, Ripoche M-A, Tost J, Journot L, Gabory A, Busato F, Le Digarcher A, Mondon F, Gut I, Jouannet $P$, et al: Modulation of imprinted gene network in placenta results in normal development of in vitro manipulated mouse embryos. Hum Mol Genet 2010, 19:1779-1790.

36. Hiby SE, Lough M, Keverne EB, Surani MA, Loke YW, King A: Paternal monoallelic expression of PEG3 in the human placenta. Hum Mol Genet 2001, 10:1093-1100.

37. Eriksson UJ: Congenital malformations in diabetic animal models-a review. Diabetes Res 1984, 1:57-66.

38. Becerra JE, Khoury MJ, Cordero JF, Erickson JD: Diabetes mellitus during pregnancy and the risks for specific birth defects: a population-based case-control study. Pediatrics 1990, 85:1-9.

39. Diamond MP, Lavy G, Polan ML: Progesterone production from granulosa cells of individual human follicles derived from diabetic and nondiabetic subjects. Int J Fertil 1989, 34:204-208.

40. Vercheval M, De Hertogh R, Pampfer S, Vanderheyden I, Michiels B, De Bernardi $P$, De Meyer R: Experimental diabetes impairs rat embryo development during the preimplantation period. Diabetologia 1990, 33:187-191

41. Shao W-J, Tao L-Y, Gao C, Xie J-Y, Zhao R-Q: Alterations in methylation and expression levels of imprinted genes $\mathrm{H} 19$ and lgf2 in the fetuses of diabetic mice. Comp Med 2008, 58:341-346.

doi:10.1186/1477-7827-11-119

Cite this article as: Ge et al:: Diabetic uterus environment may play a key role in alterations of DNA methylation of several imprinted genes at mid-gestation in mice. Reproductive Biology and Endocrinology 2013 11:119. 